\title{
ARTICLE Frequency and profile of objective cognitive deficits in hospitalized patients recovering from COVID-19
}

\author{
Abhishek Jaywant $\mathbb{D}^{1,2,3}$, W. Michael Vanderlind ${ }^{1,3}$, George S. Alexopoulos ${ }^{1,4}$, Chaya B. Fridman ${ }^{1,3}$, Roy H. Perlis ${ }^{5}$ and
} Faith M. Gunning (iD ${ }^{1,3,4}$

Early reports and case series suggest cognitive deficits occurs in some patients with COVID-19. We evaluated the frequency, severity, and profile of cognitive dysfunction in patients recovering from prolonged COVID-19 hospitalization who required acute inpatient rehabilitation prior to discharge. We analyzed cross-sectional scores from the Brief Memory and Executive Test (BMET) in a cohort of $N=57$ COVID-19 patients undergoing inpatient rehabilitation, calculating the frequency of impairment based on neuropsychologist diagnosis and by age-normed BMET subtests. In total, 43 patients (75\%) were male, 35 (61\%) were non-white, and mean age was 64.5 (SD = 13.9) years. In total, $48(84 \%)$ were previously living at home independently. Two patients had documented preexisting cognitive dysfunction; none had known dementia. Patients were evaluated at a mean of $43.2(\mathrm{SD}=19.2)$ days after initial admission. In total, 50 patients (88\%) had documented hypoxemic respiratory failure and 44 (77\%) required intubation. Forty-six patients (81\%) had cognitive impairment, ranging from mild to severe. Deficits were common in working memory (26/47 [55\%] of patients), set-shifting (21/44 [47\%]), divided attention (18/39 [46\%]), and processing speed (14/35 [40\%]). Executive dysfunction was not significantly associated with intubation length or the time from extubation to assessment, psychiatric diagnosis, or preexisting cardiovascular/metabolic disease. Attention and executive functions are frequently impaired in COVID-19 patients who require acute rehabilitation prior to discharge. Though interpretation is limited by lack of a comparator group, these results provide an early benchmark for identifying and characterizing cognitive difficulties after COVID-19. Given the frequency and pattern of impairment, easy-to-disseminate interventions that target attention and executive dysfunctions may be beneficial to this population.

Neuropsychopharmacology (2021) 46:2235-2240; https://doi.org/10.1038/s41386-021-00978-8

\section{INTRODUCTION}

Cognitive deficits are frequent, persistent, and disabling following critical illness [1, 2]. They are increasingly recognized as a common complication of COVID-19. Multiple factors associated with the illness and its treatment may contribute to cognitive sequelae. These include hypoxia, ventilation, sedation, delirium, cerebrovascular events, and inflammation [3-6]. To date, however, reports of cognitive functioning are largely limited to case reports and case series. Few investigations have used objective neuropsychological measures to quantify cognitive deficits, or to characterize the extent and profile of cognitive dysfunction during recovery from COVID-19. One existing study of 29 outpatients indicated deficits in sustained attention and reaction time [7], while another study of nine patients found impairments in global cognitive function, including attention, memory, language, and praxis [8]. The time-sensitive need to understand and address cognitive deficits early in the disease course is underscored by the prevalence of COVID-19 coupled with the long-term cognitive and psychiatric complications that were associated with the coronaviruses that caused the first Severe Acute Respiratory Syndrome (SARS) and Middle East Respiratory Syndrome (MERS) $[9,10]$.
In this study, we analyzed data from neuropsychological assessments conducted in a cohort of patients recovering from prolonged COVID-19 hospitalization who required acute inpatient rehabilitation prior to discharge. We sought to evaluate the frequency, severity, and profile of cognitive deficits in recovering, hospitalized patients with COVID-19, along with associations with mechanical ventilation, known to be linked to long-term deficits following critical illness. We explored the frequency of psychiatric diagnoses, delirium in the intensive care unit, and preexisting cardiovascular and metabolic diagnoses, and their associations with cognitive functioning.

\section{PATIENTS AND METHODS}

Sample

From April to July 2020, neuropsychological measures were administered to a cross-sectional cohort of $N=57$ hospitalized patients with COVID-19 with impairments in mobility and/or activities of daily living that necessitated acute inpatient rehabilitation prior to discharge. Rehabilitation occurred on a 22bed general inpatient rehabilitation unit with 10 overflow beds added to meet the demand and on a newly created 30-bed COVID

\footnotetext{
${ }^{1}$ Department of Psychiatry, Weill Cornell Medicine, New York, NY, US; ${ }^{2}$ Department of Rehabilitation Medicine, Weill Cornell Medicine, New York, NY, US; ${ }^{3}$ NewYork-Presbyterian Hospital/Weill Cornell Medical Center, New York, NY, US; ${ }^{4}$ Institute of Geriatric Psychiatry, Weill Cornell Medicine, White Plains, NY, US and ${ }^{5}$ Massachusetts General Hospital/ Harvard Medical School, Boston, MA, US

Correspondence: Faith M. Gunning (fgd2002@med.cornell.edu)
}

Received: 30 October 2020 Revised: 16 January 2021 Accepted: 20 January 2021

Published online: 15 February 2021 
Recovery Unit [11]. Inclusion criteria for the current analysis were as follows: (1) hospitalized for acute COVID-19 symptoms and confirmed positive for SARS-CoV-2 via polymerase chain reaction (PCR), (2) medically stable but with impairment in mobility and/or activities of daily living necessitating transfer to acute inpatient rehabilitation, (3) referred for neuropsychological evaluation for assessment of suspected cognitive dysfunction and to guide rehabilitation/discharge planning, (4) completed at least one subtest of the primary screening tool, the Brief Memory and Executive Test (BMET) described in more detail below, and (5) ability to participate in the cognitive assessment. Twenty-nine patients were not administered the BMET for the following reasons: referral question specific to psychiatric symptoms and treatment, patient not willing to participate in cognitive assessment and/or preference to focus on treatment for psychiatric symptoms (e.g., adjustment-related anxiety and depression) and/ or demoralization [12], the neuropsychologist elected to limit evaluation to psychiatric symptoms due to time constraints, or the severity of cognitive deficits precluded administration of the BMET. Data reported here were extracted from chart review after approval from the Weill Cornell Medicine Institutional Review Board with waiver of informed consent.

\section{Measures}

Demographic and clinical characteristics. Data were extracted from physician, rehabilitation therapist, social work/care manager, and neuropsychology notes in the medical record: age, gender, race/ethnicity, employment, pre-hospitalization functional level, pre-hospitalization documented cognitive dysfunction, prehospitalization cardiometabolic diagnoses (i.e., hypertension, hyperlipidemia, heart disease, stroke, sleep apnea, and diabetes), length of hospitalization in days until the neuropsychological evaluation, intubation length in days, time since extubation until the neuropsychological assessment, and presence or absence of documented delirium in the ICU. To characterize functional disability in our sample, scores were extracted from the Activity Measure for Post-Acute Care (AM-PAC Inpatient Short Form) [13] that was closest to time of admission to rehabilitation. The AMPAC is a standard-of-care instrument administered by physical and occupational therapists to assess limitations in basic activities of daily living and basic mobility.

Cognitive assessment. Patients were assessed at bedside by a clinical neuropsychologist or a neuropsychology postdoctoral fellow. Assessments occurred in a mean of 6.6 days after patients' transfer to the rehabilitation unit. Amongst cognitive domains, assessments focused on attention, executive functioning, and memory given the documented relation between these cognitive domains and functional outcome [14] and because of the relationship between these domains and inflammation, vascular processes, hypoxia, and mood and anxiety symptoms [15-18].

Data from the core neuropsychological measure, the Brief Memory and Executive Test (BMET). The BMET is comprised of multiple subtests assessing aspects of executive functioning and memory that has demonstrated strong psychometric properties and sensitivity to impairment [19]. The BMET has eight subtests that assess orientation, five-word immediate recall (working memory), five-word recall (delayed memory), five-word recognition (delayed recognition), rapid letter-number matching (divided attention), motor speed, rapid letter sequencing (visual attention and processing speed), and letter-number switching (set-shifting). The BMET letter-number switching task was substituted with the Oral Trail Making Test- $B[20,21]$ for patients who did not have adequate motor function, or for the Color Trails Test, a relatively culture-fair version of trail making, for non-English speakers [22]. Phone interpreters were relied upon to conduct evaluations for 11 patients who did not speak sufficient English to enable evaluation, and/or who preferred to communicate via an interpreter. As some subtests of the BMET were unable to be completed in all patients due to ongoing medical care/rehabilitation, and due to patient motor limitations, the number of patients completing each subtest of the BMET varied.

Psychiatric diagnosis. All patients were evaluated for symptoms of major depression and anxiety disorders, as well as adjustment to disability, by clinical interview. We extracted from the medical record the psychiatric diagnosis assigned by the neuropsychologist in the summary/impressions section of the report and related this to the presence of cognitive impairment.

\section{Statistical analysis}

Demographic and clinical characteristics were evaluated using descriptive statistics. To assess generalizability, demographic and clinical characteristics of the analyzed sample were compared to that of patients who were evaluated by neuropsychology but did not receive the BMET. We calculated the frequency of performance in the normal range, mild/borderline impaired range, and impaired range based on the normative sample and age-adjusted established cutoffs of the BMET (and Oral Trail Making Test and Color Trails Test when substitutions occurred). Impairment was classified separately for each BMET subtask. Mild/borderline was classified as a score $<1$ standard deviation below the age-adjusted normative mean, and impaired was classified as a score $<2$ standard deviation below the age-adjusted normative mean. Impairment categories were presented because of the absence of age-adjusted continuous Z-scores in the BMET normative sample. To explore the relationship between illness severity and cognition, Pearson correlations were used to compare performance on the letter-number matching (divided attention) subtest of the BMET and (1) intubation length, and (2) time between extubation and the assessment. We focused on the letter-number matching subtest because of the sensitivity of similar coding tests to executive dysfunction and functional disability [23]. Chi-square $\left(x^{2}\right)$ tests were used to explore the association between cognitive impairment and presence of pre-hospitalization cardiometabolic diagnosis, presence of delirium in the acute care/intensive care setting prior to transfer to rehabilitation, and psychiatric diagnosis.

\section{RESULTS}

Sample demographic and clinical characteristics

Approximately, $75 \%$ of the sample was male and $61 \%$ was nonwhite (Table 1). Mean age was $64.5(S D=13.9)$ years. Eighty-four percent of patients were living at home and were independent in activities of daily living prior to hospitalization. Fifty-six percent were employed. Two patients had documented cognitive difficulties prior to hospitalization; none had documented dementia. Preexisting cardiovascular and metabolic diagnoses were present in $64 \%$ of the sample. Eighty-eight percent of patients had documented hypoxia/hypoxemic respiratory failure and $77 \%$ were treated with intubation and mechanical ventilation. Twenty-nine percent of patients were weaned off ventilation using tracheostomy. At the time of admission to rehabilitation, all patients were significantly limited (mean T-scores greater than 1.5 standard deviations below normative function) in basic mobility and activities of daily living as assessed by the AM-PAC. Patients who were evaluated with the BMET did not differ from those who were not administered the BMET $(N=29)$ in age, length of hospitalization to assessment, intubation length, time from extubation to assessment, basic mobility, or activities of daily living (all $t^{\prime} s<1.59$, all $p^{\prime} s>0.11$ ). They also did not differ in proportion of gender or race/ethnicity (all $\chi^{2}<0.98$, all $p^{\prime} s>0.33$ ).

\section{Cognitive functioning}

The majority of patients (46/57 [81\%]) were assessed by neuropsychologists as having at least some degree of cognitive 
Table 1. Demographics and clinical characteristics.

\begin{tabular}{|c|c|c|}
\hline & Mean or $N(\%)$ & SD \\
\hline Age & 64.5 & 13.9 \\
\hline \multicolumn{3}{|l|}{ Gender } \\
\hline Male & $43(75 \%)$ & \\
\hline Female & $14(25 \%)$ & \\
\hline \multicolumn{3}{|l|}{ Race/Ethnicity } \\
\hline White & $22(39 \%)$ & \\
\hline Latino/Hispanic & $16(28 \%)$ & \\
\hline Black & $7(12 \%)$ & \\
\hline Asian & $11(19 \%)$ & \\
\hline Other & $1(2 \%)$ & \\
\hline \multicolumn{3}{|l|}{ Employment status } \\
\hline Employed & $32(56 \%)$ & \\
\hline Retired & $17(30 \%)$ & \\
\hline Unemployed & $5(9 \%)$ & \\
\hline Disabled & $3(5 \%)$ & \\
\hline \multicolumn{3}{|l|}{ Pre-hospitalization functional level } \\
\hline Home and independent in activities of daily living & $48(84 \%)$ & \\
\hline Home with assistance for activities of daily living & $7(12 \%)$ & \\
\hline Living in a facility & $1(2 \%)$ & \\
\hline Unknown & $1(2 \%)$ & \\
\hline \multicolumn{3}{|l|}{ Pre-hospitalization documented cognitive dysfunction } \\
\hline Known cognitive dysfunction & $2(4 \%)$ & \\
\hline No known cognitive dysfunction & $55(96 \%)$ & \\
\hline \multicolumn{3}{|c|}{$\begin{array}{l}\text { Pre-hospitalization cardiometabolic diagnoses (e.g., hypertension, hyperlipidemia, heart } \\
\text { disease, sleep apnea, stroke, and diabetes) }\end{array}$} \\
\hline Chronic cardiometabolic diagnosis & $36(64 \%)$ & \\
\hline No Diagnosis & $20(36 \%)$ & \\
\hline \multicolumn{3}{|l|}{ Language of Assessment } \\
\hline English & $46(81 \%)$ & \\
\hline Spanish & $5(9 \%)$ & \\
\hline Chinese (Mandarin, Cantonese, or regional dialect) & $4(7 \%)$ & \\
\hline Other & $2(3 \%)$ & \\
\hline Documented hypoxia/hypoxemic respiratory failure & $50(88 \%)$ & \\
\hline Intubated & $44(77 \%)$ & \\
\hline Length of intubation (days) & 13.2 & 10.1 \\
\hline Tracheostomy & $16(29 \%)$ & \\
\hline Documented delirium during acute hospitalization & $37(66 \%)$ & \\
\hline AMPAC 6-clicks basic mobility (T-score) & 34.4 & 8.6 \\
\hline AMPAC 6-clicks daily activities (T-score) & 33.8 & 6.5 \\
\hline Time from admission to assessment (days) & 43.2 & 19.2 \\
\hline Time from extubation to assessment (days) & 26.8 & 14.0 \\
\hline Time from admission to rehabilitation to assessment (days) & 6.6 & 2.6 \\
\hline \multicolumn{3}{|l|}{ Cognitive diagnosis assigned by clinician } \\
\hline Normal cognitive functioning & $11(19 \%)$ & \\
\hline Mild cognitive deficits & $27(47 \%)$ & \\
\hline Moderate cognitive deficits & $14(25 \%)$ & \\
\hline Severe cognitive deficits & $5(9 \%)$ & \\
\hline \multicolumn{3}{|l|}{ Psychiatric diagnosis assigned by clinician } \\
\hline $\begin{array}{l}\text { No diagnosis (emotional function judged to be } \\
\text { normative) }\end{array}$ & $34(60 \%)$ & \\
\hline Adjustment disorder & $13(23 \%)$ & \\
\hline Major depressive disorder & $2(3 \%)$ & \\
\hline Unspecified anxiety or mood disorder & $6(11 \%)$ & \\
\hline Preexisting psychiatric illness still present & $2(3 \%)$ & \\
\hline \multicolumn{3}{|c|}{$\begin{array}{l}\text { Values represent mean (standard deviation) for continuous measures and } \\
N(\%) \text { for categorical measures. } \\
\text { Cognitive diagnosis assigned by clinician was based on the BMET, adjunct } \\
\text { neuropsychological measures, and clinical observations. }\end{array}$} \\
\hline
\end{tabular}

deficits (Table 1). Mild cognitive impairment was most common, though moderate and severe cognitive impairment was also apparent in some patients. Four of the 57 patients were diagnosed with ongoing delirium at the time of the neuropsychological evaluation. Fig. 1 displays the percentage of patients classified as cognitively normal, mild/borderline, and impaired by subtest of the BMET, and shows that deficits were most commonly observed on subtests assessing attention and executive functions. For example, $46 \%$ of the sample (18/39 patients) exhibited impairment on the divided attention subtest. While rates of impairment on a simple motor speed task were low, impairment increased when a rapid visual attention and information processing speed component was added. Impairment increased further when a rapid set-shifting component was added. Immediate recall, which involves working memory, had greater percentage of impairment than delayed recall or delayed recognition.

Association between divided attention and intubation/extubation We calculated Z-scores for the letter-number matching test relative to the overall BMET normative sample mean and standard deviation. Because the normative sample is not separated by age, we regressed the Z-score onto age and then used the unstandardized residuals in our analysis. As shown in Fig. 2, divided attention was not significantly associated with length of intubation $(r=-0.12, p=0.49)$, or the time between extubation and assessment $(r=-0.25, p=0.19)$ after adjusting for age.

Association between cognition and psychiatric diagnosis, preexisting cardiometabolic diagnosis, and delirium

Table 1 also displays the frequency (percentage) of psychiatric diagnoses assigned by neuropsychologists. Overall, $60 \%$ of patients (34/57) did not meet criteria for a psychiatric diagnosis, which primarily included adjustment disorders. Some were assigned an unspecified mood/anxiety disorder, while a small number met clinical criteria for major depressive disorder. Using a $2 \times 2 x^{2}$ test, there was no significant relationship between cognition (normal and impaired) and psychiatric symptoms (normal and symptomatic), $X^{2}=0.92, d f=1, p=0.34$. Cognitive impairment was observed in a greater proportion of individuals with documented delirium during their acute hospitalization than those without documented delirium ( $83 \%$ vs. $63 \%$, respectively), but this difference was not statistically significant, $x^{2}=2.80, d f=1$, $p=0.09$. Likewise, there was no significant association between cognition and the presence or absence of a preexisting cardiometabolic diagnosis $\left(80 \%\right.$ vs. $75 \%$, respectively), $x^{2}=0.19$, $d f=1, p=0.67$.

\section{DISCUSSION}

In this convenience sample of medically stable and hospitalized COVID-19 patients who required acute inpatient rehabilitation prior to discharge home, 46 of 57 patients (81\%) exhibited objectively documented cognitive deficits. Attention and executive functions were most affected. That is, rate of impairment increased as tasks placed greater demands on executive functions. Divided attention, set-shifting, and processing speed had relatively high rates of impairment. Similarly, immediate recall, which places significant demands on working memory, had a high rate of impairment, whereas delayed memory and recognition memory were infrequently impaired. To our knowledge, this is among the first reports of objectively measured cognitive symptom profiles in a well-characterized sample of hospitalized individuals recovering from COVID-19.

Our findings extend those of a prior study of 29 communitydwelling adults in China recovered from COVID-19 that found a deficit in sustained attention and response time [7], though in that study the disease and treatment characteristics were not described. The early evidence in COVID-19 thus indicates that deficits in attention and executive functions are more common than deficits in memory. Although neuroimaging was not routinely performed, our findings suggest the involvement of 


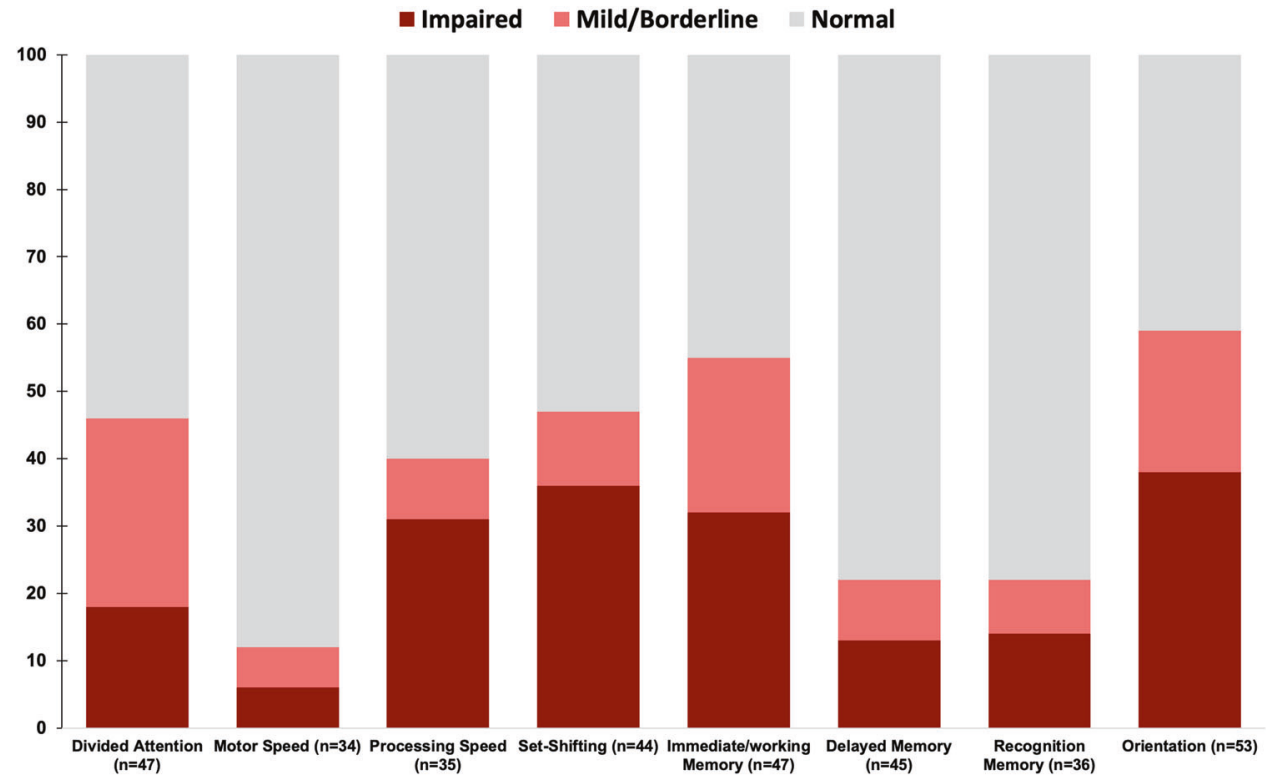

Fig. 1 Percentage impairment by subtest of the Brief Memory and Executive Test. Classification was based on published norms for the BMET, with Mild/Borderline defined as $<1$ standard deviation below the age-adjusted norms and impaired performance defined as $<2$ standard deviation below age-adjusted norms. The $x$-axis label displays the number of patients out of 57 who completed each subtest.

brain regions relevant to executive control processes including the prefrontal cortex, parietal cortex, cingulate cortex, and striatum.

We explored the association of a sensitive measure of executive function, divided attention, with intubation duration, and time between extubation and assessment. After controlling for age, divided attention was not significantly associated with either intubation duration, or time from extubation. Although longer duration of mechanical ventilation predicts worse long-term functional outcome after critical illness [24], we did not detect a significant relationship with executive dysfunction in this cohort. Intubation durations after COVID-19 have been significantly longer than in prior acute respiratory distress syndromes [25] and it is thus possible that after a certain threshold is passed by COVID-19 patients, cognitive deficits may occur irrespective of intubation duration. The absence of a positive association between time from extubation to assessment and divided attention was somewhat surprising, given that one would expect better cognitive performance further from the removal of mechanical ventilation. While it is possible that some of our patients had cognitive dysfunction due to undetected subsyndromal or residual effects of delirium, only four patients were found to have ongoing delirium at the time of the neuropsychological assessment. The high rates of orientation impairment on the BMET were predominantly due to patients incorrectly stating the exact date or floor number that may have been due to their lengthy hospitalization, rather than clinically significant disorientation. Though we cannot definitively rule out the presence of preexisting cognitive deficits, no patients in this cohort had documented preexisting dementia and the large majority were living at home without assistance for activities of daily living. Further, the presence of pre-hospitalization cardiovascular and metabolic disease was not associated with cognitive impairment, suggesting that chronic medical factors alone cannot account for our findings.

Whether the frequency and severity of cognitive deficits in COVID-19 is distinct from the known cognitive dysfunction that occurs more generally following critical illness, ICU admission, and respiratory distress remains to be determined. In (non-COVID-19) critically ill patients, delirium in the hospital occurs at a frequency of $>70 \%$ of patients [1]. At discharge, the prevalence of cognitive dysfunction is $\approx 80 \%$ for survivors of acute respiratory distress syndrome [26], which is similar to our sample in which $81 \%$ exhibited cognitive impairment. At 3 months, $40 \%$ of critically ill patients have at least mild cognitive deficits and $26 \%$ have moderate cognitive deficits [2]. Assessed at an earlier time point, on an average of 43 days after admission, we found similar percentage of COVID-19 patients with mild and moderate impairment. Our finding of predominant executive functioning deficits is also consistent with known executive dysfunction after critical illness [27]. Although there are similarities to the existing literature on critical illness, there are factors unique to COVID-19 that may increase the potential for long-term cognitive dysfunction and disability. Patients have been intubated and ventilated for long durations and cerebrovascular complications have been shown to be common [28]. Inflammatory processes, hypoxia, hypothalamicpituitary-adrenocortical axis dysregulation, coagulopathy, and organ damage may also be contributory to the observed cognitive deficits [29]. One prior study demonstrated an association between inflammation and poor sustained attention in outpatients recovered from COVID-19 [7]. The long-term evolution of cognitive deficits after COVID-19 and their unique characteristics are important questions for continued research because of the impact of cognitive dysfunction on long-term functional disability [30].

We found that $60 \%$ of the sample (34/57 patients) was assessed as having absent or normal levels of anxiety and depressed mood. Patients who were symptomatic primarily exhibited adjustmentrelated anxiety and depressed mood, while only two patients met criteria for major depressive disorder. In an exploratory analysis, cognitive impairment was not associated with psychiatric diagnosis; however, the lack of association may be affected in part by the neuropsychologists' decision not to administer the BMET to patients for whom assessing and treating psychiatric symptoms was the focus of the evaluation. After critical illness, executive functioning deficits increase risk for future depressive symptoms [31]. Thus, it will be important to examine if patients with executive dysfunction develop depressive syndromes after hospital discharge.

The generalizability of the current study is limited by the evaluation of a subset of patients undergoing acute rehabilitation in a single hospital; however, those tested with the BMET did not differ from those patients evaluated by neuropsychology who were not administered the BMET, and the demographics of our sample was consistent with known gender, racial, and ethnic 

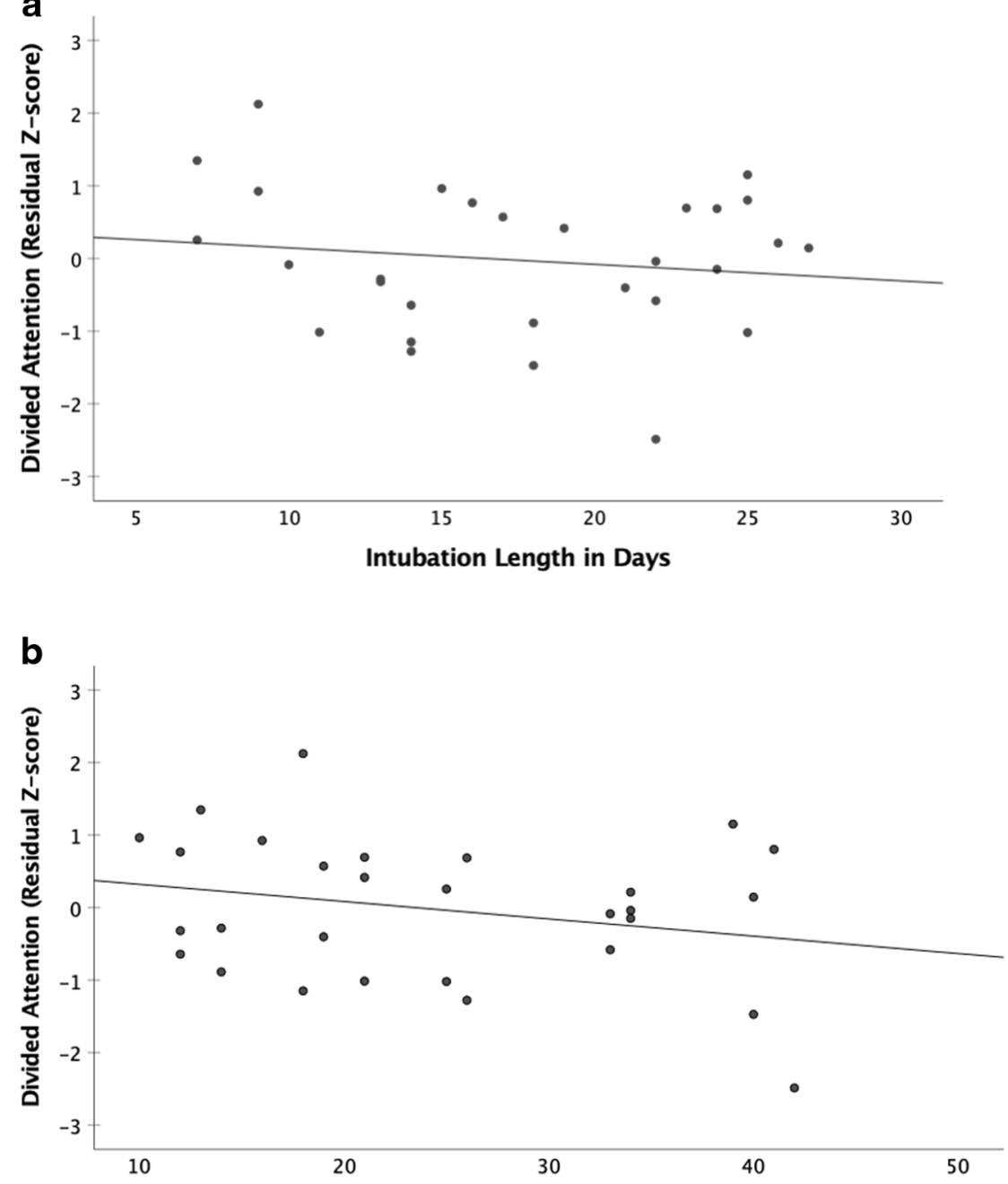

Time from Extubation to Assessment in Days

Fig. 2 Association between divided attention and a intubation length in days and $b$ time between extubation and assessment. Association between divided attention and $\mathbf{a}$ intubation length in days and $\mathbf{b}$ time between extubation and assessment. Divided Attention ( $y$ axis) is plotted as the residual Z-score relative to the normative sample after regressing out age.

differences in COVID-19 infection [32, 33]. Determining the true prevalence of cognitive dysfunction in COVID-19 will require more systematic prospective investigation. Patients referred for neuropsychological assessment may be more likely to have deficits than those who are not referred based on the observations and assessment of the referring provider, however, patients with more severe and obvious cognitive impairment are less likely to be referred for neuropsychological assessment. Our results may not generalize to patients with milder forms of COVID-19, or to those who required only brief hospitalization without acute rehabilitation.

Another limitation is the lack of a control group to which to compare our sample. Instead, our aim was to characterize cognitive symptoms in this cohort. Although we were not able to administer a comprehensive neuropsychological evaluation in the inpatient setting, the BMET enabled the quantification of the severity and pattern of deficits in aspects of attention, executive functions, and memory. Not all patients completed all subtests of the BMET, which reflects the reality of neuropsychological evaluation in a busy inpatient setting at the height of the initial surge of COVID-19 cases in New York City.

Despite these limitations, our study is among the largest to date using objective and standardized neuropsychological assessment in COVID-19 patients. Because our neuropsychologists were embedded on the rehabilitation units, we were able to administer in-person cognitive assessments, while following designated infection control procedure. Our results may help to establish a benchmark for early cognitive dysfunction after severe COVID-19 illness.

In summary, we show that medically stable inpatients who were recovering from prolonged COVID-19 hospitalization and required acute inpatient rehabilitation prior to discharge, commonly have impairments in attention and executive functions including working memory, divided attention, and set-shifting. The frequency of impairment was not related to the presence of chronic cardiovascular and metabolic disease. Divided attention was not associated with intubation duration or time from extubation to assessment. While intubation duration may be one measure of a more severe course, other markers of illness severity may be associated with cognitive sequelae. Our results provide an early benchmark for studying the evolution of cognitive difficulties in recovering COVID-19 patients. They also highlight the importance of studying interventions that target attention and executive functioning after COVID-19. Given the prevalence of COVID-19, targeting these deficits through scalable cognitive interventions that have been demonstrated to improve 
similar deficits and can be widely disseminated in patients' homes through reliance on technology [34] may support optimal cognitive and functional outcomes.

\section{FUNDING AND DISCLOSURE}

The authors did not receive any funding to complete this study. None of the authors have competing financial interests or conflicts related to this study. GSA has served on the speakers' bureaus of Allergan, Otsuka, and Takeda-Lundbeck and on advisory groups for Janssen and Eisai. RHP has received consulting fees for service as a scientific advisor to Belle.ai, Burrage Capital, Genomind, Outermost Therapeutics, RID Ventures, and Takeda. He holds equity in Belle.ai, Outermost Therapeutics, and Psy Therapeutics. $A J, W M V, C B F$, and FMG have no additional interests to declare.

\section{ACKNOWLEDGEMENTS}

We thank Ruchi Patel, MA OTR/L, Gargi Doulatani, BA, and Karen Wen, BA, for their assistance in data extraction and management. We are grateful for the tireless physicians, nurses, and rehabilitation therapists who worked with us to assist COVID19 survivors in their rehabilitation and recovery.

\section{AUTHOR CONTRIBUTIONS}

AJ conducted clinical interviews and neuropsychological evaluations, extracted and verified the data, conducted data analyses, and wrote the manuscript. WMV conducted clinical interviews and neuropsychological evaluations, extracted and verified the data, and edited the manuscript. GSA and CBF provided input in measure selection, interpretation of results, and manuscript writing. RHP and FMG assisted in data analysis, interpretation of results, and manuscript writing. All authors read and approved the manuscript.

\section{ADDITIONAL INFORMATION}

Publisher's note Springer Nature remains neutral with regard to jurisdictional claims in published maps and institutional affiliations.

\section{REFERENCES}

1. Girard TD, Thompson JL, Pandharipande PP, Brummel NE, Jackson JC, Patel MB, et al. Clinical phenotypes of delirium during critical illness and severity of subsequent long-term cognitive impairment: a prospective cohort study. Lancet Respir Med 2018;6:213-22.

2. Pandharipande PP, Girard TD, Jackson JC, Morandi A, Thompson JL, Pun BT, et al. Long-term cognitive impairment after critical illness. N. Engl J Med 2013;369: 1306-16.

3. Helms J, Kremer S, Merdji H, Schenck M, Severac F, Clere-Jehl R, et al. Delirium and encephalopathy in severe COVID-19: A cohort analysis of ICU patients. Crit Care 2020;24:1-11.

4. Varatharaj A, Thomas N, Ellul MA, Davies NWS, Pollak TA, Tenorio EL, et al. Neurological and neuropsychiatric complications of COVID-19 in 153 patients: a UK-wide surveillance study. The Lancet. Psychiatry 2020;7:875-82.

5. Romero-Sánchez C, Díaz-Maroto I, Fernández-Díaz E, Sánchez-Larsen Á, LayosRomero A, García-García J, et al. Neurologic manifestations in hospitalized patients with COVID-19: the ALBACOVID registry. Neurology. 2020;95:e1060-e1070.

6. Beach SR, Praschan NC, Hogan C, Dotson S, Merideth F, Kontos N, et al. Delirium in COVID-19: A case series and exploration of potential mechanisms for central nervous system involvement. Gen Hosp Psychiatry 2020;65:47-53.

7. Zhou H, Lu S, Chen J, Wei N, Wang D, Lyu H, et al. The landscape of cognitive function in recovered COVID-19 patients. J Psychiatr Res 2020;129:98-102.

8. Negrini F, Ferrario I, Mazziotti D, Berchicci M, Bonazzi $M$, de Sire A, et al. Neuropsychological features of severe hospitalized COVID-19 patients at clinical stability and clues for post-acute rehabilitation. Arch Phys Med Rehabil. 2020. 2020. https://doi.org/10.1016/j.apmr.2020.09.376.

9. Rabinovitz B, Jaywant A, Fridman C. Neuropsychological functioning in severe acute respiratory disorders caused by the coronavirus: implications for the current COVID-19 pandemic. Clin Neuropsychol. 2020;34:1453-79.

10. Sheng B, Cheng SKW, Kwok KL, Ho LL, Chan ELY. The effects of disease severity, use of corticosteroids and social factors on neuropsychiatric complaints in severe acute respiratory syndrome (SARS) patients at acute and convalescent phases. Eur Psychiatry 2005;20:236-42.

11. Gupta R, Gupta A, Ghosh AK, Stein J, Lindsay L, Beckley A, et al. A Paradigm for the Pandemic: A Covid-19 Recovery Unit. NEJM Catal Innov Care Deliv. 2020. 2020. https://doi.org/10.1056/CAT.20.0238.

12. Jaywant $A$, Vanderlind WM, Boas SJ, Dickerman AL Behavioral interventions in acute COVID-19 recovery: a new opportunity for integrated care. Gen Hosp Psychiatry. 2020. 2020. https://doi.org/10.1016/j.genhosppsych.2020.07.001.

13. Jette DU, Stilphen M, Ranganathan VK, Passek SD, Frost FS, Jette AM. Validity of the AM-PAC '6-clicks' inpatient daily activity and basic mobility short forms. Phys Ther 2014:94:379-91.

14. Cahn-Weiner DA, Boyle PA, Malloy PF. Tests of executive function predict instrumental activities of daily living in community-dwelling older individuals. Appl Neuropsychol 2002;9:187-91.

15. Uiterwijk R, van Oostenbrugge RJ, Huijts $M$, De Leeuw PW, Kroon AA, Staals J. Total cerebral small vessel disease MRI score is associated with cognitive decline in executive function in patients with hypertension. Front Aging Neurosci 2016;8:1-8.

16. Trollor JN, Smith E, Agars E, Kuan SA, Baune BT, Campbell L, et al. The association between systemic inflammation and cognitive performance in the elderly: the Sydney memory and ageing study. Age (Omaha) 2012;34:1295-308.

17. Culley DJ, Snayd M, Baxter MG, Xie Z, Lee IH, Rudolph J, et al. Systemic inflammation impairs attention and cognitive flexibility but not associative learning in aged rats: possible implications for delirium. Front Aging Neurosci 2014;6:1-7.

18. Garcia-Molina A, Roig-Rovira T, Enseñat-Cantallops A, Sanchez-Carrion R, PicoAzanza N, Bernabeu M, et al. Neuropsychological profile of persons with anoxic brain injury: differences regarding physiopathological mechanism. Brain Inj 2006;20:1139-45.

19. Brookes RL, Hollocks MJ, Khan U, Morris RG, Markus HS. The Brief Memory and Executive Test (BMET) for detecting vascular cognitive impairment in small vessel disease: a validation study. BMC Med 2015;13:1-8.

20. Mrazik M, Millis S, Drane DL. The oral trail making test: effects of age and concurrent validity. Arch Clin Neuropsychol 2010;25:236-43.

21. Jaywant A, Barredo J, Ahern DC, Resnik L. Neuropsychological assessment without upper limb involvement: a systematic review of oral versions of the Trail Making Test And Symbol-digit Modalities Test. Neuropsychol Rehabil. 2016;28: 1055-77.

22. D'Elia LF, Satz P, Uchiyama CL, White T color trails test. Lutz, FL: psychological assessment resources; 1996.

23. Jaywant A, Toglia J, Gunning FM, O'Dell MW. The clinical utility of a 30-minute neuropsychological assessment battery in inpatient stroke rehabilitation. J Neurol Sci 2018;390:54-62.

24. Wilson ME, Barwise A, Heise KJ, Loftsgard TO, Dziadzko M, Cheville A, et al. Longterm return to functional baseline after mechanical ventilation in the ICU. Crit Care Med 2018;46:562-9.

25. Zilberberg MD, Nathanson BH, Ways J, Shorr AF. Characteristics, hospital course, and outcomes of patients requiring prolonged acute versus short-term mechanical ventilation in the United States, 2014-8. Crit Care Med 2020;48: 1587-94.

26. Honarmand K, Lalli RS, Priestap F, Chen JL, Mclntyre CW, Owen AM, et al. Natural history of cognitive impairment in critical illness survivors a systematic review. Am J Respir Crit Care Med 2020;202:193-201.

27. Sukantarat KT, Burgess PW, Williamson RCN, Brett SJ. Prolonged cognitive dysfunction in survivors of critical illness. Anaesthesia 2005;60:847-53.

28. Ntaios G, Michel P, Georgiopoulos G, Guo Y, Li W, Xiong J, et al. Characteristics and outcomes in patients with COVID-19 and acute ischemic stroke: the global COVID-19 stroke registry. Stroke 2020;51:e254-e258.

29. ladecola C, Anrather J, Kamel H. Effects of COVID-19 on the nervous system. Cell 2020;183:16-27.

30. Norman BC, Jackson JC, Graves JA, Girard TD, Pandharipande PP, Brummel NE, et al. Employment outcomes after critical illness: an analysis of the bringing to light the risk factors and incidence of neuropsychological dysfunction in ICU survivors cohort. Crit Care Med 2016;44:2003-9.

31. Duggan MC, Wang L, Wilson JE, Dittus RS, Ely EW, Jackson JC. The relationship between executive dysfunction, depression, and mental health-related quality of life in survivors of critical illness: Results from the BRAIN-ICU investigation. J Crit Care 2017;37:72-9.

32. Webb Hooper M, Napoles AM, Perez-Stable EJ COVID-19 and racial/ethnic disparities. JAMA. 2020. 2020. https://doi.org/10.1001/jama.2020.8598.

33. Jin JM, Bai P, He W, Wu F, Liu XF, Han DM, et al. Gender differences in patients with COVID-19: focus on severity and mortality. Front Public Heal 2020;8:1-6.

34. Bove RM, Rush G, Zhao C, Rowles W, Garcha P, Morrissey J, et al. A videogamebased digital therapeutic to improve processing speed in people with multiple sclerosis: a feasibility study. Neurol Ther 2019;8:135-45. 